\title{
Current Perspectives on Corneal Transplantation (Part 2)
}

\author{
Yee Ling Wong $\mathbb{D}^{\prime}$, Siyin Liu' ${ }^{1,2}$, Andrew Walkden $\mathbb{1}^{1,2}$ \\ 'Manchester Royal Eye Hospital, Manchester University NHS Foundation Trust, Manchester, UK; ${ }^{2}$ School of Biological Sciences, Faculty of Biology, \\ Medicine and Health, University of Manchester, Manchester, UK \\ Correspondence: Andrew Walkden, Manchester Royal Eye Hospital, Oxford Road, Manchester, MI3 9WL, UK, Email walkdenandrew@gmail.com
}

\begin{abstract}
Disease of the cornea is the third leading cause of blindness worldwide. Corneal graft surgery is one of the most successful forms of solid organ transplantations in humans, with ever increasing developments in surgical technique. To date, approximately 4504 corneal transplants are performed in the UK each year. While full thickness transplantation was the most commonly performed keratoplasty over the last few decades, selective lamellar transplantation of the diseased layers of the cornea has been universally adopted. This comprehensive review aims to provide an updated synthesis on different types of corneal transplantations, their treatment outcomes, and the associated complications of each procedure both in adult and pediatric populations. In addition, we also present an up-to-date summary of the emerging therapeutic approaches that have the potential to reduce the demand for donordependent keratoplasty.
\end{abstract}

Keywords: corneal transplantation, corneal blindness, keratoplasty, graft rejection, graft survival

\section{Pediatric Penetrating Keratoplasty}

Corneal transplantation remains the treatment of choice for the management of potentially amblyogenic corneal stromal opacities, edema or severe ectasia within the pediatric population to ensure adequate visual development. It is, however, highly challenging due to the high risk of graft failure and vascularization when compared to adult keratoplasty, resulting in poorer visual outcomes. ${ }^{1,2}$ The reported reasons for worse visual outcomes include associated comorbidities such as anterior segment dysgenesis, congenital cataracts, optic nerve insults or hypoplasia, all of which limit visual potential and result in a guarded preoperative visual prognosis. Increased fibrinous reactions have been reported postoperatively making it more likely that graft rejection will occur. ${ }^{1,3,4}$ Furthermore, the amblyogenic potential of preoperative corneal opacities and postoperative astigmatism can severely hinder visual development, and can lead to disappointing visual outcomes postoperatively despite a clear corneal graft. ${ }^{2}$ When considering pediatric keratoplasty, a complete ocular examination, usually under general anesthesia is required to evaluate for potential comorbidities and visual outcomes for the patient, usually with concurrent ultrasonography or OCT evaluation. Postoperative management requires adequate education and "buy-in" of the involved carers in order to optimize long-term visual potential alongside amblyopia management. ${ }^{1}$

\section{Indication}

Due to the rare indications for pediatric keratoplasty only 5\% of all corneal transplants in Australia from 1985 to 2009 and approximately 1 in 24,000 live births in the US. ${ }^{3,5}$ Indication varies by region - congenital corneal opacities such as Peters Anomaly are the most common indication for keratoplasty in North America, anterior segment dysgenesis in Asia, infectious keratitis or corneal scarring in Africa and traumatic corneal scars in Northern Europe. ${ }^{1,6}$ Other indications for pediatric corneal transplants include keratoconus, trauma, chemical burns, and nontherapeutic corneal scars, which can also occur in adulthood. ${ }^{4,6}$ 


\section{Graft Survival and Outcomes by Indication}

Keratoconus is one of the main indications for penetrating keratoplasty in childhood. ${ }^{8}$ The reported outcomes within the literature are good, with $80-100 \%$ graft survival at five years. ${ }^{4,6,7}$ Graft survival in the large Australian Corneal Graft Registry Study by Lowe et al showed favorable outcomes in corneal transplants, with $75 \%$ of recipients aged between 13 and 19 years old achieving BCVA of 0.5 on logMAR, with survival rates of more than $90 \%$ at 10 years postoperatively. ${ }^{4,5}$ A clinical outcome study in Denmark by Hovlykke et al shows that the best postoperative BCVA was achieved in the keratoconus subgroup with $86 \%$ patients having better BCVA compared to preoperatively. ${ }^{9}$

Despite the potential difficulties with pediatric corneal transplants, it can be argued that the period gained with visual stimuli and subsequent visual pathway development can ultimately improve overall perception, growth and development of a child if the intervention is early enough. ${ }^{9}$ The reasons for high failure rates are cited as lax corneoscleral tissue, a more aggressive inflammatory response; intracameral and ocular surface response, robust wound healing and persistent intraocular inflammation. Other factors also include ocular surface instability, glaucoma, and the risk of amblyopia in visual rehabilitation. ${ }^{9}{ }^{10}$ Hovlykke et al described six primary graft failure cases in their study, where short-term graft failure was mainly caused by infection (52\%; herpes related $25 \%$, corneal ulcer nonherpes related $27 \%$ ) or vascularization $(41 \%)$. Long-term failure was due to endothelial failure.

The indications for pediatric corneal transplants vary throughout the literature, and range between 14 and $64 \%$ for congenital reasons, 19 and $80 \%$ for acquired nontraumatic reasons and 6 and 29\% for acquired traumatic reasons. Hovlykke et al demonstrates that grafts in patients treated for keratoconus (92\%) and stromal dystrophies (100\%) have the highest survival rates at median follow-up times of 15-20 years. ${ }^{9}$ In contrast, grafts treated for acquired traumatic corneal opacities have poor survival rates (failure rate of $63 \%$ ). As expected, regraft procedures have failure rates as high as $80 \% .{ }^{9}$ A 40 -year retrospective cohort study by Majander et al reported all cases of grafts in aniridic patients failed to remain clear. Only two out of six grafts undertaken for congenital corneal opacities remained clear. Nonetheless, the only eye grafted for herpetic keratitis-related corneal scarring retained its clarity for at least 25 years postoperatively with BCVA of $0.3 .{ }^{6}$ Children requiring a transplant following a herpetic keratitis generally have a good prognosis with graft survival rates of more than $80 \%$ with a median follow-up duration of 2.7 years. ${ }^{7}$ A multicenter retrospective study looking at ocular trauma consisting of children aged 12 years and younger by Dana et al, however, showed a favorable overall success in penetrating keratoplasty in children comparable to adults, with a graft survival rate of $84 \%$ at one year and $70 \%$ at two years or more if the injury did not involve the posterior segment. Graft survival falls to less than onethird in trauma cases involving the posterior segment. In terms of complications, no cases of endophthalmitis have been reported. 8(36\%) eyes experienced at least one rejection episode, three treated successfully. Four $(50 \%)$ occurred within the first six months, all within two years of transplantation. Five out of six failed due to graft rejection, and one because of phthisis. This study shows that if there is only the anterior segment involvement, one can expect a favorable outcome if penetrating keratoplasty is done in a timely manner, coupled with visually appropriate visual rehabilitation to reduce the risk of amblyopia. ${ }^{10}$

Corneal opacities of the cornea due sclerocornea and Peters Anomaly are often managed with penetrating keratoplasty at an early stage to allow visual development at infancy and childhood to prevent deprivational amblyopia. ${ }^{7,11}$ Anterior segment dysgenesis is a congenital disorder arising from the neural crest during and manifest as a spectrum of disorders during the development of the anterior segment. Both sclerocornea and Peters Anomaly share common clinical features such as anterior synechiae, posterior embryotoxon, prominent iris process and congenital glaucoma. Peters Anomaly is different in comparison to sclerocornea in that in some cases the patient has a clear peripheral cornea with scleral tissue extension and corneal vascularization. ${ }^{11}$ Most studies have report a poor prognosis in patients with sclerocornea, with graft survival said to be in the region of $12-30 \%$ with a follow-up duration of five years. Peters Anomaly also has a poor prognosis requiring multiple corneal transplants, which is further complicated by secondary glaucoma. ${ }^{7,11-14}$ The risk of graft failure in Peters Anomaly is high, up to $39 \%$ at one year, $38 \%$ at three years, and $70 \%$ at five years. ${ }^{4,15,16} \mathrm{Kim}$ et al evaluated the survival rates and median survival times of corneal grafts in patients aged five or younger with either sclerocornea or Peters Anomaly. The results show that only half of the grafts survived at follow-up of $92.7 \pm 10$ months and five years postoperatively. Mean survival time was 135.6 \pm 17.9 months in patients with Peters Anomaly and 36.4 
\pm 16.1 months in sclerocornea $(p=0.014)$. Survival rate was $87.5 \%$ in Peters Anomaly and $25.0 \%$ in sclerocornea $(p=0.02)$. They also found that presence of vascularization and smaller donor corneas were associated with higher risk of graft failure. ${ }^{11}$ In terms of visual outcomes, Lin et al looked at patients aged 0 to 7 who has undergone transplant surgery for a congenital corneal opacity and found that patients with unilateral sclerocornea had the worst visual outcome. Retransplantation and lensectomy were associated with poorer visual outcomes, however in the presence of lens-corneal adhesion or lens dysplasia, lensectomy is unavoidable in some cases. ${ }^{17}$

Another important determining factor appears to be the age of the child at time of surgery. It has been reported that younger patients tend to have poorer visual outcomes. Lowe et al reported poorer graft survival in infants younger than five years old, when compared to a group of children aged 5 to 12 years old and teenagers. ${ }^{1,5}$ In congenital corneal opacities requiring corneal transplants, $50 \%$ corneal grafts survived in those five years old or younger. ${ }^{11}$ Gulias-Cañizo et al also found significant association between graft rejection and age $\left(\mathrm{r}=0.153 ; \mathrm{R}^{2}=0.023 ; p=0.048\right)$ with mean age of rejection at $10.84+4.8$ years. ${ }^{4}$

\section{Conclusion}

The survival of grafts in the pediatric population is not as promising as that in adult patients and is largely dependent upon the age of the patient, preexisting ocular comorbidities and the primary indication for corneal transplant. ${ }^{6,7}$ However, with the advances in surgical techniques and more effective pre- and postoperative management, the survival rate for pediatric keratoplasty has improved over the years. A multidisciplinary approach in the management of pediatric keratoplasty and cooperation with parents are extremely important in determining visual outcomes.

\section{Pediatric Lamellar Keratoplasty}

Lamellar Keratoplasty has gained popularity among the pediatric population due to the inherent advantages over primary penetrating keratoplasty. Anterior lamellar (superficial anterior lamellar keratoplasty, SALK and DALK) procedures reduce the risk of graft rejection and help to maintain structural integrity whereas posterior lamellar procedures (DSAEK and DMEK) are associated with improved safety profile, accelerated wound healing, minimal astigmatism hence could potentially provide a better visual rehabilitation for the amblyogenic pediatric population. Of all pediatric corneal transplants, lamellar keratoplasties constitute about $28 \%$, which are performed in conditions such as congenital hereditary endothelial dystrophy (CHED), congenital glaucoma (CG), keratomalacia, anterior segment developmental anomalies, iris cyst, rubella syndrome, and corneal trauma. ${ }^{18}$

\section{Indications}

Pediatric SALK has been performed for superficial corneal opacities due to previous keratitis, shield ulcers, congenital dermoid and Salzmann nodular degeneration, and corneal dystrophies. ${ }^{18,19}$ DALK has shown to be an effective modality for conditions such as keratoconus, microbial keratitis, corneal scarring, exposure keratopathy, dermoid, corneal dystrophies, MPS, SJS, ocular rosacea and chemical injury. ${ }^{20-23}$ In PLK, DSAEK have been indicated in CHED, polymorphous posterior corneal dystrophy (PPMD), Peters Anomaly, decompensated corneas, buphthalmos, traumatic aniridia and rejected PKP grafts. ${ }^{18}$ DMEK however, have been performed in endothelial dysfunction in PPCD and Kearns-Sayre syndrome. ${ }^{24,25}$

\section{Outcomes and Complications SALK}

According to Watts et al, BCVA of $\geq 6 / 12$ were seen in $86.7 \%$ of the cases in 48 pediatric eyes who underwent SALK for limbal dermoid at 12 months. They noted microperforations in $6.3 \%$ of the cases, of which regrafts were indicated in two patients. Other complications include graft melt (2\%), deep vascularization (23\%), mild corneal opacification (26\%) and suture granuloma (3.3\%). ${ }^{26}$ Guber et al compared 12-year outcomes of pediatric SALK with adult SALK. Data suggests good visual outcomes when combined with good amblyopic therapy-mean VA pediatric vs adult ( 0.45 vs 0.55 $\log$ MAR). Nonetheless, pediatric SALK required more intensive follow up due to higher complication rates (56\% vs $38 \%$ ) and need for surgical revisions ( $43 \%$ vs $11 \%){ }^{25,27}$ 
DALK

Ashar et al reported graft clarity in $69.2 \%$ pediatric patients $(n=26)$ at 7.8 years follow-up following a DALK indicated for anterior stromal opacity. ${ }^{21}$ Arora et al reported graft clarity of $90 \%$ at 44.5 months follow-up following a DALK indicated for advanced keratoconus without hydrops. ${ }^{28}$ Elbaz et al reported $96 \%$ graft clarity at 42.7 months in MPS. Nonetheless $17.6 \%$ had intraoperative microperforation although none of them required conversion to $\mathrm{PK}^{22} \mathrm{DM}$ detachments were seen in 7.6\% (2/26) cases (Ashar et al) vs $0.05 \%(1 / 20)$ cases (Arora et al). ${ }^{21,28}$ The majority of these occurred intraoperatively and were managed conservatively. Arora et al reported one case (5\%) of stromal rejection and Elbaz et al reported five cases $(9.8 \%)$ of stromal rejection following a DALK. ${ }^{22,28}$ Other complications include unstable refraction, high astigmatism, suture related complications and Urrets-Zavalia syndrome. ${ }^{18}$

\section{DSAEK}

The rates of graft detachment following a DSAEK can be as high as $21 \%$ due to lack of postoperative supine posturing in children, uncontrolled eye rubbing and anatomically rough retrocorneal surface or retained DM. ${ }^{18,29}$ ECL post DSAEK ranges from $19-53 \% .{ }^{29-31}$ Lenticular opacification can be as high as $40 \%$ post DSAEK due to intra- and postoperative insults from lens touch, prolonged air tamponade, repeated surgical interventions and prolonged use of topical steroid. Other complications include rise of IOP (pupillary block at the immediate postoperative phase and steroid response at the later stage), graft rejection, persistent stromal haze and progressive anterior segment fibrosis. In terms of visual outcomes, the optical clarity may not be as good as PK due to interface irregularities. Poor visual gain may be contributed by residual corneal haze, increased corneal thickness and amblyopia. ${ }^{18}$

\section{DMEK}

It is difficult to ascertain long-term outcomes of DMEK in children due to the limited data available. Ali et al report nine eyes (mean age 5.8 \pm 3.7 years) that underwent DMEK for endothelial dystrophies. Postoperatively, DMEK graft detachment was seen in five eyes, graft failure related to graft dislocation was seen in two eyes and primary endothelial failure was seen in one eye. There were no cases of elevated IOP and graft rejection. Mean VA improvement was 0.50 \pm 0.14 from $0.88 \pm 0.22$ at 12 months. Overall graft survival rate was $66.7 \% .{ }^{32}$ Gonnermann et al also reported a case of successful DMEK in a 12-year-old child with corneal decompensation, with a resultant decrease in corneal thickness from 837 to $583 \mu \mathrm{m}$ at six-month follow-up. ${ }^{33}$

\section{Conclusion}

Although LK has many advantages compared to PK, long term outcomes especially in DSAEK and DMEK grafts in the pediatric population are difficult to ascertain. Careful considerations should be given to the risks of repeated general anesthesia for postoperative complications and surgical interventions, unpredictable visual outcomes and long-term monitoring.

\section{The Boston Keratoprosthesis}

The introduction of keratoprosthesis (artificial corneal implantation) has brought about a paradigm shift in the future of corneal transplants. Dohlman et al first described the original collar-button shaped Boston keratoprosthesis (BKPro) prototype made of polymethyl methacrylate (PMMA) in the 1970s. The outcomes of keratoprosthesis from the first published paper were mostly unfavorable. Nonetheless, subsequent modification and scientific advancement of keratoprosthesis led to the FDA approval of the device in 1992 and 19,000 devices have been implanted as of the year 2019. ${ }^{34-36}$ The BKPro is available with customized aphakic powers for various axial lengths and also for standard pseudophakic powers. Parts of the BKPro consist of a front plate, a central stem consisting of a locking interface, and a back plate which is now available in two materials - the original PMMA and a newer titanium model which has been reported to be superior in terms of strength, resistance to corrosion and less fibrogenic nature. ${ }^{37-39}$ The surgical technique of the original type 1 BKPro include preparation of an $8.5 \mathrm{~mm}-9.0 \mathrm{~mm}$ donor corneal button, a $3 \mathrm{~mm}$ central trephination before outer diameter punch for better centration, placing the donor button onto the stem of the front plate and placing the back place onto this complex. ${ }^{40-42}$ Lens extraction is necessary at the time of BKPro implantation in a phakic eye. In a pseudophakic eye 
however, depending on the stability of the intraocular lens, the lens can be left in place or explanted. Core anterior vitrectomy is normally performed in an aphakic eye during BKPro implantation. ${ }^{40}$

\section{Indication}

Traditionally, BKPro was indicated for bilateral severe corneal blindness due to multiple failed corneal transplants and for eyes that have a poor prognosis for penetrating keratoplasty especially those with ocular surface disease such as mucous membrane pemphigoid and SJS. Over the years, its use has extended to include other conditions such as neurotrophic corneas, herpetic keratitis, aniridia, pediatric corneal opacities, autoimmune ocular disorders, extensive corneal neovascularization, chemical injuries and stem cell deficiency. ${ }^{37,40}$ Patient selection for BKPro implantation is important as patients require lifelong follow-up. Those who are known to have poor compliance to medications and attendance should not be considered for BKPro implantation. Patients with a healthy contralateral eye with reasonable visual function (20/50 or better) should be dissuaded from keratoprosthesis surgery as the diseased eye should be kept in reserve if needed in the future. In addition, patients with preexisting conditions like end-stage glaucoma, severe optic neuropathy, chronic retinal detachment and macular pathology would not benefit from BKPro implantation as the risks would outweigh the potential visual benefits. It is also important to ensure patients have sufficient tear production in order to keep the donor corneal component functioning. Certain pathologies such as SJS and MMP that result in an arid ocular surface do not always do well with BKPro implantation for this reason. ${ }^{40}$

The visual outcomes of keratoprosthesis however, vary significantly and are related to the indication for implantation and concurrent pathology. According to a large case series from Yaghouti et al, the most favorable outcomes are in patients with graft failure in a noncicatrizing conditions (68\% have BCVA between 0 and 1.00 logMAR at five years), reasonable outcomes in those with ocular cicatricial pemphigoid (43\% at five years) and chemical burns (64\% at five years) with the worst outcomes being in Steven-Johnson syndrome (SJS, $0 \%$ at five years). ${ }^{43}$

\section{PK vs BKPro for Corneal Graft Failure}

Ahmad et al published a systematic review and meta-analysis to evaluate the clinical outcomes of repeat PK vs BKPro implantation. The pooled data shows that the probability of achieving BCVA 1.0 logMAR or better is higher with a BKPro compared to a repeat PK at two years (80\%; 95\%CI: 68-88\% vs $42 \%$; 95\%CI: 30-56\%). Visual prognosis remains higher with BKPro at 37 months follow-up duration. The retention rate of BKPro was $98.9 \%, 94 \%$ and $75 \%$ at one, two, and five years, respectively (95\%CI: 64-84\%) compared to 79\%, 67\%, and 47\% (95\%CI: 40-54\%) survival rate for clear grafts. Their results also show $25 \%(95 \% \mathrm{CI}: 10-44 \%)$ an incidence of glaucoma requiring intervention after a repeat PK compared to $30.7 \%$ following a BKPro at three years. In cases of repeat PK, intraocular pressure (IOP) rise is likely due to the closed-angle mechanism and steroid use. In contrast, in patients with a BKPro, conventional and accurate IOP measurements are difficult due to the prosthetic ocular surface, and can present challenges in the management of glaucoma. The rate of infectious keratitis was $18 \%$ in PK compared to $2.9 \%$ in BKPro at five years. Incidence of endophthalmitis in BKPro implantation was 5.4\% in literature between years 2001 and 2011. This meta-analysis demonstrates superiority of BKPro implantation in visual outcomes as compared to PK in patients with previously failed PKs, however this needs to be balanced against the risk of both glaucoma, retinal detachment and endophthalmitis. It is also important to consider that, if BKPro implantation fails requiring explantation, severe visual loss could be irreversible. $^{44}$

\section{BKPro as Primary Penetrating Corneal Procedure}

A retrospective interventional comparative study compared patients with BKPro as a primary procedure (group 1) with patients who had PK prior to BKPro (group 2). A single surgeon based in Canada carried out all procedures. The results show that $87 \%$ patients from group 1 and $63 \%$ from group 2 have BCVA of $1.0 \log$ MAR at 12 months $(p=0.03)$. Eightseven percent of eyes from group 1 and $80 \%$ from group 2 have an improvement of two lines or more during the followup period. Four (13\%) eyes from group 1 and $15(38 \%)$ eyes from group 2 failed to achieve vision of $1.0 \operatorname{logMAR}$ at 12 months due to preoperative comorbidities like glaucoma and postoperative complications such as, corneal melt (3.3\% vs $5.0 \% p=1.00)$, endophthalmitis $(3.3 \%$ vs $2.5 \% p=1.00)$, retinal detachment $(20.0 \%$ vs $37.5 \% p=0.19)$ and choroidal 
hemorrhage ( 13.3 vs $7.5 \%, p=0.45)$. More common postoperative complications include postoperative glaucoma $(66.7 \%$ vs $75 \% p=0.59)$, retroprosthetic membrane (RPM) formation ( $33.3 \%$ vs $37.5 \% p=0.80)$, and persistent inflammation $(30.0 \%$ vs $37.5 \% p=0.61)$ in both groups. ${ }^{45} \mathrm{RPM}$ formation is a fairly common complication occurring up to $25-65 \%$ cases within a year of BKPro implant, of which $45 \%$ would eventually require treatment in the form of YAG membranotomy and $11 \%$ requiring surgical revision. ${ }^{46}$ The manufacturer's use of a titanium back plate was intended to reduce the risk of RPM formation. ${ }^{47}$ There was no significant difference in complications or retention rates in both groups. 28 (93\%) eyes from group 1 and $36(90 \%)$ from group 2 had at least one postoperative complication. There were no intraoperative complications in both groups. This study reported favorable outcomes at 12 months postoperatively in respect to visual outcomes, complications and retention rates. BKPro have shown to be effective in cases especially where PKs are expected to fail, for example, severe limbal stem cell deficiency, neurotrophic keratopathy and aniridia. ${ }^{45}$

\section{BKPro in Challenging Cases}

De la Paz et al presented an interesting study describing the outcome of patients with BKPro in challenging cases (chemical or thermal burn vs autoimmune vs other diagnoses) in regard to anatomical and visual outcomes. They included 12 eyes with chemical and thermal burns, 16 eyes with autoimmune diseases (SJS, Lyell's syndrome, graft versus host disease (GVHD), ocular cicatricial pemphigoid and immunotactoid keratopathy), six eyes with bullous keratopathy, five eyes with congenital aniridia keratopathy, seven eyes with leukoma following keratitis, four eyes with ocular trauma, three eyes with congenital opacities, three eyes with corneal ectatic disease, one eye with calcific keratopathy and one eye with limbal stem cell deficiency due to contact lens overuse, one eye with cicatricial trachoma and one eye with congenital glaucoma. Patients younger than 12 years old were excluded from data analysis. In terms of anatomical survival rates, the outcome of the autoimmune cases was better than those with chemical or thermal burns, but was similar to those in the "other diagnoses". From three years onwards, there was a tendency for the chemical or thermal burn group to worsen but the other two groups remained stable $(p=0.26)$. In terms of visual acuity, the chemical or thermal burn and the autoimmune groups had better BCVA results one year postoperatively. At three years, BCVA tends to worsen in the autoimmune and "other diagnoses" group. Their results show very good anatomical and visual outcomes at three years postoperatively in the chemical or thermal burn group. In their paper, they reported ocular cicatricial pemphigoid as an absolute contraindication for BKPro as the presence of severe dry eye disease, symblepharon and ocular fibrosis would make bandage contact lens nonadaptable Instead of BKPro, a suitable alternative would be biological keratoprosthesis like osteo-odonto-keratoprosthesis (OOKP) or tibial bone KPro. ${ }^{48}$ A prospective study by Magalhães et al also supports the use of BKPro in managing bilateral limbal stem cell deficiency secondary to ocular injury with good anatomical and functional results. They also suggested BKPro as an alternative for visual rehabilitation in patients with severe ocular surface disease as the majority (90\%) of patients have significant improvement in BCVA up to two years postoperatively. Care should be taken as persistent epithelial defects are common complications postoperatively and carry an increased risk of sterile stromal necrosis. ${ }^{49}$

\section{Management of BKPro}

Postoperatively, a soft bandage contact lens will be placed over the keratoprosthesis indefinitely and replaced regularly. This allows for better hydration of exposed tissue, enhancing the device retention and such to reduce the risk of dellen formation, epithelial defects and corneal melt. Patients will also require a topical regimen of steroids tapered according to the levels of inflammation. Use of broad-spectrum topical antibiotics prophylactically which include topical fluoroquinolone drops with an addition of $1.4 \%$ vancomycin have significantly reduced the incidence of endophthalmitis. ${ }^{34,37,40,50}$

\section{Osteo-odonto-keratoprosthesis (OOKP)}

OOKP was first described in Rome by Professor Benedetto Strampelli, where a support for an artificial cornea from the patient's own tooth root and surrounding alveolar bone are used to provide support for an artificial corneal device to restore cornea related visual loss not amenable to other forms of surgery. It is based on the principle using a patient's own tooth as a biological frame to support an acrylic optic in patients with end-stage ocular surface disease. ${ }^{51,52}$ Falcinelli further improved the pioneering work by Strampelli in a stepwise fashion. This is to ensure long-term visual outcome and 
retention. ${ }^{53,54}$ The indications of OOKP include bilateral blindness due to SJS, ocular cicatricial pemphigoid, Lyell's syndrome/TENS, epidermolysis bullosa acquisita, trachoma, chemical injury and physical injury, lid loss (Crouzon disease), vascularized corneas with complete limbal stem cell deficiency, multiple failed penetrating keratoplasty and corneal decompensation following vitrectomy with silicone oil filling that cannot be removed safely. On the contrary, patients younger than 17 years old, eyes with no perception of light (NPL), phthisis, and those with posterior segment pathologies that severely affect visual potential are all absolute contraindications to OOKP. Flash-VEP and flash-ERG are useful to identify retinal function but may not be reliable to predict visual outcomes due to masking of amplitudes due to significant anterior segment scarring. It is also important to discuss, the acceptance of cosmetic appearance, potential complications, follow-up, compliance and patient expectations postoperatively. ${ }^{4,55}$

\section{Outcomes}

OOKP has demonstrated to be dependable with an anatomical retention of above $80 \%$ over a period of 20 years. It has proven to be rewarding to many patients with end stage blindness, with more than half of patients achieving BCVA of better than $0.3 \log$ MAR ${ }^{56,57}$ A $100 \%$ survival rate was seen in series published by Tan et al, Marchi et al, and Hille et al at five-year follow-up. ${ }^{57-59}$ In all studies reviewed in the case series by Tan et al, the anatomical survival was $87.8 \%$ (range 67-100\%; SD 0.15) at five years in all studies and 81.0\% (range 65-98\%; SD 0.16) in three studies at 20 years. In terms of visual outcomes, $52 \%$ (range $46-72 \%$; SD 0.22) of patients achieved BCVA better than 0.48 logMAR. Eyes requiring regrafts where the entire optical cylinder and lamina had to be removed and replaced with either another OOKP or a tectonic graft occurred in 1-5 patients across studies reviewed. 4-11 patients throughout the studies had mucosal repeat grafts for lamina extrusion or mucous membrane ulceration. One to twelve patients had a repeat mucosal clearance for overgrowth over the optical cylinder. Three to ten patients required glaucoma surgery and three to four patients required a vitrectomy. ${ }^{57}$

\section{Complications}

The most common complication occurring up to $50 \%$ include buccal mucosal graft issues such as mucous membrane overgrowth occluding the optical cylinder, ulceration, or exposure of lamina. ${ }^{60,61}$ Mucosal overgrowth can be managed by trimming and cautery. Mitomycin $\mathrm{C}$ can be used in recurrent cases, but as an off-label application. Mucosal ulcerations, however, are managed according to their severity and underlying cause, that is, ischemia, dryness, or infection. For small ulcers, extensive lubrication may be adequate, however for larger ulcers, patients may require surgical intervention with tarsal pedicle, mucosal rotation, buckle handle flaps and free patch grafts. Glaucoma is an important risk factor for poor visual outcome despite a good anatomical result for OOKP. Difficulties in measuring the patient's IOP further complicate the monitoring of glaucoma and this relies on manual digital palpation. Furthermore, anti-glaucoma medications are often not absorbed through the thick mucosal membrane graft. ${ }^{60}$ A paper published by Falcinelli et al reported the prevalence of preexisting glaucoma of $36.4 \%$ in 181 patients, whereas Zarei-Ghanavati et al reported prevalence of de novo glaucoma of $15 \%$ and preexisting glaucoma of $40 \% .{ }^{60,62}$ Intracapsular lens extraction during OOKP may decrease the incidence of glaucoma, allowing optic nerve assessments to take place using optical coherence tomography and visual field testing. Management would include systemic acetazolamide, sublingual timolol or off-label beta-blockers. Surgical intervention may be required to reduce IOP. In terms of retinal and choroidal complications, vitreous hemorrhage and choroidal detachments are usually self-resolving in the early postoperative period ${ }^{60}$ Only one-third of patients require vitreoretinal input for either diagnosis or management of complications. ${ }^{63}$ Nonetheless, retinal detachment repair has a low success rate of only $20 \%$ thus it is important to educate patients regarding signs and symptoms of conditions such as retinal detachment and endophthalmitis where timely treatment improves outcomes. ${ }^{64}$

\section{CorNeat KPro}

The CorNeat KPro is a novel device using nanoscale chemical engineering technology that creates a scaffold that stimulates cellular growth and subsequent invasion of native cells. It comprises a central PMMA optical member and an external integrating skirt consisting of carbonated polyurethane fibers and is placed under the conjunctiva. Its unique features allow short- and long-term integration, and animal (rabbit) studies have shown high $(87.5 \%)$ retention rate with 
diffuse infiltration of fibroblast with collagen deposition among the fibrils composing the kPro implant. This resolves the issues of tissue availability seen in BKPro. The results of the study are promising, as all implants were retained with no hypotony at one month postoperatively. Nonetheless, lenticular opacities were noted in five out of eight implanted eyes likely due to lens touch perioperatively. This can be explained by the different ocular anatomy where the anterior chamber is narrower in rabbit eyes. Subacute bacterial endophthalmitis was reported in one case seven weeks postoperatively. ${ }^{65}$ A prospective, open label, single arm study is currently recruiting human patients to assess the safety and efficacy of CorNeat KPro for the treatment of corneal blindness. ${ }^{66}$

\section{Future Perspectives}

\section{Application of Femtosecond-lasers in Corneal Transplantation Surgery}

Currently the most well-known ophthalmic application of femtosecond-lasers is corneal flap creation during laser refractive surgery such as laser-assisted in-situ keratomileusis. Recent advances in the use of femtosecond-laser assisted keratoplasty (FLAK) has been an exciting development in corneal transplantation methods. The precise computer-guided cutting in FLAK enables incisions of different configurations, such as zig-zag, top hat, mushroom, and decagonal shapes. ${ }^{67}$ The increased surface area and interlocking surfaces between graft and host tissues allows for faster wound healing. Various studies have reported that FLAK has improved visual outcome with better BCVA and lower astigmatism compared with conventional PK. ${ }^{68-70}$

There are many theoretical advantages of the femtosecond laser-assisted DALK (FSDALK) over its manual version. Computer-guided placement of femtosecond-lasers can induce incisions in the cornea at precise location and depth. In DALK, separation of DM from host stroma is notoriously the most difficult and crucial step. If unsuccessful, DM perforation may occur and lead to conversion to PK, even in experienced hands. Creating a trephination and a deep lamellar incision with femtosecond-laser were shown to increase the chance of complete removal of the overlying stroma and the baring of the host DM. ${ }^{71,72}$ Multiple studies have demonstrated that FSDALK provides superior conformation of the donor tissue to the host bed, allowing faster healing, earlier suture removal, ${ }^{73,74}$ lower rejection rate, ${ }^{75}$ and enhanced visual outcomes. ${ }^{69,73}$ One safety concern of FSDALK is femtosecond-laser induced endothelial cell death if the incision is placed too close to the DM. Although very few studies have specifically investigated endothelial cell loss after FSDALK, Li et al reported no difference in 12-month ECD loss between eyes that underwent FSDALK and DALK. ${ }^{73}$

\section{DM Stripping-Only without Endothelial Keratoplasty (DWEK)}

Over the past decade, different types of endothelial keratoplasties had largely succeeded PK as the mainstream technique to manage FECD. These techniques are associated with issues inherent to corneal transplantations, such as graft rejection, postkeratoplasty complications (eg glaucoma, graft detachment, primary graft failure), and tissue availability. Inspired by the observation of corneal clearance after iatrogenic trauma, ${ }^{76-79}$ "descemetorhexis without endothelial keratoplasty" (DWEK) is a procedure that involves the removal of a small area of the failed central endothelium and DM without transplantation of a donor corneal graft, thus reducing the reliance on the limited donor pool. ${ }^{80}$ The procedure has also been termed "descemetorhexis without graft placement/grafting", "DM stripping-only without endothelial keratoplasty (DSO)". ${ }^{81,82}$ Earlier observations on DWEK reported mixed results after stripping DM with a diameter of 6-9 mm in clearing corneal edema $(0-66.7 \%),{ }^{83-85}$ while smaller descemetorhexis of 3-4 mm appeared to provide superior clearance rates (63.2-100\%). ${ }^{81,86-88}$ Persistent non-clearing corneal edema may need to undergo endothelial keratoplasty. ${ }^{83,84}$ Potential complications associated with DWEK include posterior stromal scarring possibly due to unpredictable healing responses initiated by intraoperative trauma to the stroma, ${ }^{89} \mathrm{DM}$ detachment (which may require re-bubbling), ${ }^{81}$ and irregular astigmatism. ${ }^{82}$ Regarding postoperative visual outcomes, the reported BCVA ranged from -0.12 to $0.13 \log$ MAR with variable follow-up time (4-36 months). ${ }^{81,86}$ Huang et al reported no significant difference between postoperative BCVA in DWEK and DMEK cases (0.16 vs $0.13 \log$ MAR), although the DWEK cases took longer to reach $0.3 \log$ MAR vision (7.1 weeks vs DMEK 2.2 weeks) ${ }^{87}$ 


\section{Tissue Engineered Corneas}

Due to the worldwide mismatch between demand and supply of human corneal tissue, bioengineered cornea is an exciting prospect to help overcome the problems associated with insufficient donor supply. The corneal tissue-equivalent should ideally mimic native tissue both structurally and functionally. ${ }^{90}$ The construct needs to support the growth of a functional corneal epithelial sheet for protection of intraocular contents from pathogenic invasion; the mechanical strength of the scaffold should be comparable to the stiffness of native corneal stroma; the microscopic collagen fibrillar structure of the construct needs to be parallel as in natural stroma in order to achieve optical transparency. ${ }^{90}$ To create tissue-equivalent with these properties, attempts have been made on decellularized xenogeneic tissues ${ }^{91,92}$ and natural or synthetic polymers ${ }^{93-95}$ with various level of success. More evidence is required to confirm the efficacy of these approaches.

\section{Emerging Therapies for Endothelial Dysfunction}

As mentioned above, the shortage of corneal grafts is a significant limitation on corneal transplantations. This is a particularly challenging problem in a society with aging populations and an increased prevalence of corneal endothelial diseases like FCED. Rho-kinase (ROCK) signalling is an important cellular pathway that plays a role in a wide spectrum of pathological conditions. ${ }^{96}$ ROCK inhibitor (Y-27632) was found to enhance corneal endothelial cell proliferation, promote cell adhesion, and suppressed apoptosis. ${ }^{97}$ In vivo studying using ROCK inhibitor eye drops on animal models with a damaged corneal endothelium demonstrated the promotion of wound healing. ${ }^{98,99}$ Early clinical studies investigating the effect of ROCK inhibitors on patients with FCED revealed various levels of success. ${ }^{99,100}$ Topical administration of ROCK-inhibitor has been found to be able to promote endothelial cell migration and salvage failing DWEK cases. ${ }^{81}$ More recently, Macsai et al compared the outcomes of patients who underwent DWEK with topical use of ROCK-inhibitor postoperatively against those who underwent DWEK alone, and they found that the ROCK-inhibitortreated group experienced a significantly faster visual recovery (4.6 vs 6.5 weeks) and had higher central endothelial cell counts (1086 vs 736 cells $/ \mathrm{mm}^{2}$ ). ${ }^{88}$ Further clinical trials with larger sample sizes are required to confirm the efficacy of ROCK inhibitor eye drops.

Cell therapy appears to have the potential to replace deficient tissues. Researchers have managed to directly expand corneal endothelial cells in vitro from cells extracted from human corneal grafts. ${ }^{101}$ Recently, the simultaneous injections of cultured human CECs and ROCK-inhibitor after DSO has also demonstrated improved CEC density (mean final CEC density 1257 cells $/ \mathrm{mm}^{2}$ ) and visual acuity (mean final BCVA $0.05 \operatorname{logMAR}$ ) in 10 of 11 treated eyes after five-year follow-up. ${ }^{102}$ Another strategy is autologous stem cell-based therapies, which use adult stem cells such as skin-derived precursors, mesenchymal stem cells, and induced pluripotent stem cells for the expansion of corneal endothelial cells. ${ }^{103}$ Bioengineered materials have also been developed to aid the expansion of endothelial cells and eventual delivery of cultured cell sheet by intracameral injection. ${ }^{104}$

Preclinical studies investigating gene-based approaches that utilize antisense oligonucleotides (ASO) have yielded promising effects on ameliorating the cellular toxicity mediated by the trinucleotide repeat expansion on the gene TCF4, a gene that is known to cause FECD, in patient-derived CEC models. ${ }^{105,106}$ These efforts have culminated in first-in-man clinical trials. ${ }^{107,108}$ Further clinical trials is required to determine to safety and efficacy of ASO as a gene-based therapy for FECD.

\section{Conclusion}

A century has passed since the first corneal transplant was performed, and the evolution within the field of corneal transplantation has been extraordinary. While full thickness replacement has been the most popular form of corneal transplantation for decades, lamellar keratoplasties only targeting affected layers of tissues have quickly gained popularity owing to their superior visual outcome and enhanced graft survival rate. Technological and scientific advancements continue to foster developments in laser-assisted keratoplasty, tissue engineered cornea and cell therapies, which further drive the evolution in treatment of corneal diseases. Exciting emerging therapies have the potential to offer specific cellular replacement of the affected areas, and could eventually replace conventional keratoplasty in certain conditions. 


\section{Disclosure}

SL is supported by the National Institute of Health Research (ACF-2019-06-009). The authors report no other conflicts of interest in this work.

\section{References}

1. Trief D, Marquezan MC, Rapuano CJ, Prescott CR. Pediatric corneal transplants. Curr Opin Ophthalmol. 2017;28(5):477-484. doi:10.1097/ ICU.0000000000000393

2. Al-Ghamdi A, Al-Rajhi A, Wagoner MD. Primary pediatric keratoplasty: indications, graft survival, and visual outcome. JAAPOS. 2007;11 (1):41-47. doi:10.1016/j.jaapos.2006.09.012

3. Aquavella JV. Incidence of peters anomaly and congenital opacity. Cornea. 2015;34(2):e4. doi:10.1097/ICO.0000000000000316

4. Gulias-Cañizo R, Gonzalez-Salinas R, Hernandez-Zimbron LF, Hernandez-Quintela E, Sanchez-Huerta V. Indications and outcomes of pediatric keratoplasty in a tertiary eye care center: a retrospective review. Medicine. 2017;96(45):e8587. doi:10.1097/MD.0000000000008587

5. Lowe MT, Keane MC, Coster DJ, Williams KA. The outcome of corneal transplantation in infants, children, and adolescents. Ophthalmology. 2011;118(3):492-497. doi:10.1016/j.ophtha.2010.07.006

6. Majander A, Kivelä TT, Krootila K. Indications and outcomes of keratoplasties in children during a 40-year period. Acta Ophthalmol. 2016;94 (6):618-624. doi:10.1111/aos.13040

7. Lang SJ, Böhringer D, Reinhard T. Keratoplastik bei Kindern. [Keratoplasty in Children]. Ophthalmologe. 2020;117(3):215-217. doi:10.1007/ s00347-019-01006-3

8. Cowden JW. Penetrating keratoplasty in infants and children. Ophthalmology. 1990;97(3):324-329. doi:10.1016/S0161-6420(90)32586-1

9. Hovlykke M, Hjortdal J, Ehlers N, Nielsen K. Clinical results of 40 years of paediatric keratoplasty in a single university eye clinic. Acta Ophthalmol. 2014;92(4):370-377. doi:10.1111/aos.12198x

10. Dana M-R, Schaumberg DA, Moyes AL, et al. Outcome of penetrating keratoplasty after ocular trauma in children. Arch Ophthalmol. 1995;113 (12):1503-1507. doi:10.1001/archopht.1995.01100120033003

11. Kim YW, Choi HJ, Kim MK, Wee WR, Yu YS, Oh JY. Clinical outcome of penetrating keratoplasty in patients 5 years or younger: Peters anomaly versus sclerocornea. Cornea. 2013;32(11):1432-1436. doi:10.1097/ICO.0b013e31829dd836

12. Lang SJ, Richter C, Richter O, Böhringer D, Geerling G, Reinhard T. Keratoplastiken im Kindes- und Jugendalter. [Keratoplasty in Children and Adolescence]. Klin Monbl Augenheilkd. 2018;235(03):319-323. doi:10.1055/s-0042-124512

13. Gupta S, Khurana A. Comment on "visual outcomes and prognostic factors of successful penetrating keratoplasty in 0- to 7-year-old children with congenital corneal opacities. Cornea. 2019;38(4):e10. doi:10.1097/ICO.0000000000001860

14. Yang LLH, Lambert SR, Lynn MJ, Stulting RD. Long-term results of corneal graft survival in infants and children with peters anomaly. Ophthalmology. 1999;106(4):833-848. doi:10.1016/S0161-6420(99)90175-6

15. Dana MR, Schaumberg DA, Moyes AL, et al. Corneal transplantation in children with peters anomaly and mesenchymal dysgeneses. Ophthalmology. 1997;104(10):1580-1586. doi:10.1016/S0161-6420(97)30093-1

16. Chang JW, Kim MK, Kim JH, Kim S-J, Wee WR, Yu YS. Long-term visual outcomes of penetrating keratoplasty for Peters anomaly. Graefes Arch Clin Exp Ophthalmol. 2013;251(3):953-958. doi:10.1007/s00417-012-2165-7

17. Lin Q, Shi W, Miao S, Zhang Y, Li L, Pan Z. Visual outcomes and prognostic factors of successful penetrating keratoplasty in 0- to 7-year-old children with congenital corneal opacities. Cornea. 2018;37(10):1237-1242. doi:10.1097/ICO.000000000001689

18. Sharma N, Agarwal R, Jhanji V, Bhaskar S, Kamalakkannan P, Nischal KK. Lamellar keratoplasty in children. Surv Ophthalmol. 2020;65 (6):675-690. doi:10.1016/j.survophthal.2020.04.002

19. Fogla R, Knyazer B. Microkeratome-assisted two-stage technique of superficial anterior lamellar keratoplasty for Reis-Bücklers corneal dystrophy. Cornea. 2014;33(10):1118-1122. doi:10.1097/ICO.0000000000000189

20. Chew ACY, Mehta JS, Tan DTH. Deep lamellar keratoplasty after resolution of hydrops in keratoconus. Cornea. 2011;30(4):454-459. doi:10.1097/ICO.0b013e3181f0b1f3

21. Ashar JN, Pahuja S, Ramappa M, Vaddavalli PK, Chaurasia S, Garg P. Deep anterior lamellar keratoplasty in children. Am J Ophthalmol. 2013;155(3):570-574.e1. doi:10.1016/j.ajo.2012.09.029

22. Elbaz U, Kirwan C, Shen C, Ali A. Avoiding big bubble complications: outcomes of layer-by-layer deep anterior lamellar keratoplasty in children. Br J Ophthalmol. 2018;102(8):1103-1108. doi:10.1136/bjophthalmol-2017-310962

23. Harding SA, Nischal KK, Upponi-Patil A, Fowler DJ. Indications and outcomes of deep anterior lamellar keratoplasty in children. Ophthalmology. 2010;117(11):2191-2195. doi:10.1016/j.ophtha.2010.03.025

24. Dua HS, Faraj LA, Said DG, Gray T, Lowe J. Human corneal anatomy redefined. Ophthalmology. 2013;120(9):1778-1785. doi:10.1016/j. ophtha.2013.01.018

25. Sharma N, Maharana P, Singhi S, Aron N, Patil M. Descemet stripping automated endothelial keratoplasty. Indian J Ophthalmol. 2017;65 (3):198. doi:10.4103/ijo.IJO_874_16

26. Watts P, Michaeli-Cohen A, Abdolell M, Rootman D. Outcome of lamellar keratoplasty for limbal dermoids in children. JAAPOS. 2002;6 (4):209-215. doi:10.1067/mpa.2002.124651

27. Guber I, Bergin C, Othenin-Girard P, Munier F, Majo F. 12-year outcomes of microkeratome-assisted anterior lamellar therapeutic keratoplasty (ALTK) for disorders of the anterior part of the corneal stroma - a comparative review of adult and children. Klin Monatsbl Augenheilkd. 2018;235(04):404-408. doi:10.1055/s-0044-101828

28. Arora R, Jain P, Jain P, Manudhane A, Goyal J. Results of deep anterior lamellar keratoplasty for advanced keratoconus in children less than 18 years. Am J Ophthalmol. 2016;162:191-198.e2. doi:10.1016/j.ajo.2015.11.020

29. Madi S, Santorum P, Busin M. Descemet stripping automated endothelial keratoplasty in pediatric age group. Saudi J Ophthalmol. 2012;26 (3):309-313. doi:10.1016/j.sjopt.2012.04.006

30. Busin M. Descemet-stripping automated endothelial keratoplasty for congenital hereditary endothelial dystrophy. Arch Ophthalmol. 2011;129 (9):1140. doi:10.1001/archophthalmol.2011.114 
31. Price MO, Price DA, Fairchild KM, Price FW. Rate and risk factors for cataract formation and extraction after Descemet stripping endothelial keratoplasty. Br J Ophthalmol. 2010;94(11):1468-1471. doi:10.1136/bjo.2009.175174

32. Ali A, Woo JH, Rootman D, Mireskandari K. Outcomes of descemet membrane endothelial keratoplasty in children with endothelial dystrophies. Invest Ophthalmol Vis Sci. 2020;61(7):3561.

33. Gonnermann J, Klamann MKJ, Maier A-KB, et al. Descemet membrane endothelial keratoplasty in a child with corneal endothelial dysfunction in Kearns-Sayre syndrome. Cornea. 2014;33(11):1232-1234. doi:10.1097/ICO.0000000000000252

34. Saeed HN, Shanbhag S, Chodosh J. The Boston keratoprosthesis. Curr Opin Ophthalmol. 2017;28(4):390-396. doi:10.1097/ ICU.0000000000000373

35. Dohlman CH, Schneider HA, Doane MG. Prosthokeratoplasty. Am J Ophthalmol. 1974;77(5):694-700. doi:10.1016/0002-9394(74)90534-0

36. Chodosh J. FDA approval obtained for the Boston keratoprosthesis type I Lucia design. 2019:12.

37. Nonpassopon M, Niparugs M, Cortina MS. Boston type 1 keratoprosthesis: updated perspectives. OPTH. 2020;14:1189-1200. doi:10.2147/ OPTH.S219270

38. Tan XW, Riau A, Shi ZL, et al. In vitro effect of a corrosive hostile ocular surface on candidate biomaterials for keratoprosthesis skirt. $B r J$ Ophthalmol. 2012;96(9):1252-1258. doi:10.1136/bjophthalmol-2012-301633

39. Dong Y, Yang J, Wang L, et al. An improved biofunction of Titanium for keratoprosthesis by hydroxyapatite-coating. ELT J. 1991;28:252-259.

40. Magalhães FP, de Sousa LB, de Oliveira LA. Boston type I keratoprosthesis: review. Arq Bras Oftalmol. 2012;75(3):218-222. doi:10.1590/ S0004-27492012000300016

41. Khalifa YM, Moshirfar M. Improved centration of the type 1 Boston Keratoprosthesis in donor carrier tissue. OPTH. 2010;4:931-933. doi: $10.2147 / \mathrm{OPTH} . \mathrm{S} 12778$

42. Zerbe BL, Belin MW, Ciolino JB. Results from the multicenter Boston type 1 keratoprosthesis study. Ophthalmology. 2006;113(10):1779-1784 e1. doi:10.1016/j.ophtha.2006.05.015

43. Yaghouti F, Nouri M, Abad JC, Power WJ, Doane MG, Dohlman CH. Keratoprosthesis: preoperative prognostic categories. Cornea. 2001;20 (1):19-23. doi:10.1097/00003226-200101000-00003

44. Ahmad S, Mathews PM, Lindsley K, et al. Boston type 1 keratoprosthesis versus repeat donor keratoplasty for corneal graft failure: a systematic review and meta-analysis. Ophthalmology. 2016;123(1):165-177. doi:10.1016/j.ophtha.2015.09.028

45. Fadous R, Levallois-Gignac S, Vaillancourt L, Robert M-C, Harissi-Dagher M. The Boston Keratoprosthesis type 1 as primary penetrating corneal procedure. Br J Ophthalmol. 2015;99(12):1664-1668. doi:10.1136/bjophthalmol-2014-306161

46. Stacy RC. Characterization of retrokeratoprosthetic membranes in the Boston type 1 keratoprosthesis. Arch Ophthalmol. 2011;129(3):310. doi:10.1001/archophthalmol.2011.26

47. Todani A, Ciolino JB, Ament JD, et al. Titanium back plate for a PMMA keratoprosthesis: clinical outcomes. Graefes Arch Clin Exp Ophthalmol. 2011;249(10):1515-1518. doi:10.1007/s00417-011-1684-y

48. de La Paz MF, Stoiber J, de Rezende Couto Nascimento V, et al. Anatomical survival and visual prognosis of Boston type I keratoprosthesis in challenging cases. Graefes Arch Clin Exp Ophthalmol. 2014;252(1):83-90. doi:10.1007/s00417-013-2481-6

49. Magalhães FP, Hirai FE, de Sousa LB, de Oliveira LA. Boston type 1 keratoprosthesis outcomes in ocular burns. Acta Ophthalmol. 2013;91(6): e432-e436. doi:10.1111/aos.12083

50. Williamson SL, Cortina MS. Boston type 1 keratoprosthesis from patient selection through postoperative management: a review for the keratoprosthetic surgeon. Clin Ophthalmol. 2016;10:437-443. doi:10.2147/OPTH.S83677

51. Strampelli B. Keratoprosthesis with osteodontal tissue. Am J Ophthalmol. 1963;89:1029-1039.

52. Liu C, Paul B, Tandon R, et al. The Osteo-Odonto-Keratoprosthesis (OOKP). Semin Ophthalmol. 2005;20(2):113-128. doi:10.1080/ 08820530590931386

53. Falcinelli G, Missiroli A, Petitti V. Osteo-odonto-keratoprosthesis up-to-date. Acta XXV Concil Ophthalmol Milan. 1987;2:2772-2776.

54. Falcinelli G, Barogi G, Taloni M. Osteoodontokeratoprosthesis: present experience and future prospects. Refract Corneal Surg. $1993 ; 9: 193$.

55. Hille K, Grabner G, Liu C, et al. Standards for Modified Osteoodontokeratoprosthesis (OOKP) surgery according to Strampelli and Falcinelli: the Rome-Vienna protocol. Cornea. 2005;24(8):895-908. doi:10.1097/01.ico.0000157401.81408.62

56. Avadhanam VS, Liu CSC. A brief review of Boston type-1 and osteo-odonto keratoprostheses. Br J Ophthalmol. 2015;99(7):878-887. doi:10.1136/bjophthalmol-2014-305359

57. Tan A, Tan DT, Tan X-W, Mehta JS. Osteo-odonto keratoprosthesis: systematic review of surgical outcomes and complication rates. Ocul Surf. 2012;10(1):15-25. doi:10.1016/j.jtos.2012.01.003

58. Marchi V, Ricci R, Pecorella I, Ciardi A, Di Tondo U. Osteo-odonto-keratoprosthesis: description of surgical technique with results in 85 patients. Cornea. 1994;13(2):125-130. doi:10.1097/00003226-199403000-00004

59. Hille K, Hille A, Ruprecht KW. Medium term results in keratoprostheses with biocompatible and biological haptic. Graefes Arch Clin Exp Ophthalmol. 2006;244(6):696-704. doi:10.1007/s00417-005-0092-6

60. Zarei-Ghanavati M, Avadhanam V, Vasquez Perez A, Liu C. The osteo-odonto-keratoprosthesis. Curr Opin Ophthalmol. 2017;28(4):397-402. doi: $10.1097 / \mathrm{ICU} .0000000000000388$

61. Basu S, Pillai VS, Sangwan VS. Mucosal complications of modified osteo-odonto keratoprosthesis in chronic Stevens-Johnson syndrome. Am J Ophthalmol. 2013;156(5):867-873.e2. doi:10.1016/j.ajo.2013.06.012

62. Falcinelli G. Modified osteo-odonto-keratoprosthesis for treatment of corneal blindness: long-term anatomical and functional outcomes in 181 cases. Arch Ophthalmol. 2005;123(10):1319. doi:10.1001/archopht.123.10.1319

63. Lim LS, Ang CL, Wong E, Wong DWK, Tan DTH. Vitreoretinal complications and vitreoretinal surgery in osteo-odonto-keratoprosthesis surgery. Am J Ophthalmol. 2014;157(2):349-354. doi:10.1016/j.ajo.2013.08.033

64. Hughes EH, Mokete B, Ainsworth G, et al. Vitreoretinal complications of osteoodontokeratoprosthesis surgery. Retina. 2008;28(8):1138-1145. doi:10.1097/IAE.0b013e318174e10e

65. Litvin G, Klein I, Litvin Y, Klaiman G, Nyska A. CorNeat KPro: ocular implantation study in rabbits. Cornea. 2021;40(9):1165-1174 doi:10.1097/ICO.0000000000002798

66. ClinicalTrials.gov. First in human (FIH) study to assess safety and efficacy of the CorNeat KPro for the treatment of corneal blindness. Available from: https://clinicaltrials.gov/ct2/show/NCT04485858. Accessed September 5, 2021. 
67. Chamberlain WD. Femtosecond laser-assisted deep anterior lamellar keratoplasty. Curr Opin Ophthalmol. 2019;30(4):256-263. doi:10.1097/ ICU.0000000000000574

68. Chamberlain WD, Rush SW, Mathers WD, Cabezas M, Fraunfelder FW. Comparison of femtosecond laser-assisted keratoplasty versus conventional penetrating keratoplasty. Ophthalmology. 2011;118(3):486-491. doi:10.1016/j.ophtha.2010.08.002

69. Shehadeh-Mashor R, Chan C, Yeung SN, Lichtinger A, Amiran M, Rootman DS. Long-term outcomes of femtosecond laser-assisted mushroom configuration deep anterior lamellar keratoplasty. Cornea. 2013;32(4):390-395. doi:10.1097/ICO.0b013e318254a4e4

70. Wade M, Muniz Castro H, Garg S, et al. Long-term results of femtosecond laser-enabled keratoplasty with zig-zag trephination. Cornea. 2019;38(1):42-49. doi:10.1097/ICO.0000000000001783

71. Farid M, Steinert RF. Deep anterior lamellar keratoplasty performed with the femtosecond laser zigzag incision for the treatment of stromal corneal pathology and ectatic disease. J Cataract Refract Surg. 2009;35(5):809-813. doi:10.1016/j.jcrs.2009.01.012

72. Price FW, Price MO, Grandin JC, Kwon R. Deep anterior lamellar keratoplasty with femtosecond-laser zigzag incisions. $J$ Cataract Refract Surg. 2009;35(5):804-808. doi:10.1016/j.jcrs.2009.01.011

73. Li S, Wang T, Bian J, Wang F, Han S, Shi W. Precisely controlled side cut in femtosecond laser-assisted deep lamellar keratoplasty for advanced keratoconus. Cornea. 2016;35(10):1289-1294. doi:10.1097/ICO.0000000000000962

74. Alio JL, Abdelghany AA, Barraquer R, Hammouda LM, Sabry AM. Femtosecond laser assisted deep anterior lamellar keratoplasty outcomes and healing patterns compared to manual technique. Biomed Res Int. 2015;2015:397891. doi:10.1155/2015/397891

75. Gonzalez A, Price MO, Feng MT, Lee C, Arbelaez JG, Price FW. Immunologic rejection episodes after deep anterior lamellar keratoplasty: incidence and risk factors. Cornea. 2017;36(9):1076-1082. doi:10.1097/ICO.0000000000001223

76. Srivastava V, Singh A, Chowdhary R. Spontaneous resolution of corneal oedema after inadvertent descemetorhexis during cataract surgery. Med $J$ Armed Forces India. 2010;66(2):177-179. doi:10.1016/S0377-1237(10)80142-X

77. Choo SY, Zahidin AZM, Then KY. Re: spontaneous corneal clearance despite graft detachment in Descemet membrane endothelial keratoplasty. Am J Ophthalmol. 2010;149(3):531. doi:10.1016/j.ajo.2009.11.010

78. Pan JC-H, Au Eong K-G. Spontaneous resolution of corneal oedema after inadvertent "descemetorhexis" during cataract surgery. Clin Exp Ophthalmol. 2006;34(9):896-897. doi:10.1111/j.1442-9071.2006.01360.x

79. Baráková D, Darsová D. A case report illustrating the spontaneous vision recovery after inadvertent central descemetorhexis during anterior capsulotomy. IMCRJ. 2021;14:45-49. doi:10.2147/IMCRJ.S290179

80. Kaufman AR, Nosé RM, Pineda R. Descemetorhexis Without Endothelial Keratoplasty (DWEK): proposal for nomenclature standardization. Cornea. 2018;37(4):e20-e21. doi:10.1097/ICO.0000000000001528

81. Moloney G, Petsoglou C, Ball M, et al. Descemetorhexis without grafting for fuchs endothelial dystrophy—supplementation with topical ripasudil. Cornea. 2017;36(6):642-648. doi:10.1097/ICO.0000000000001209

82. Iovieno A, Neri A, Soldani AM, Adani C, Fontana L. Descemetorhexis without graft placement for the treatment of fuchs endothelial dystrophy: preliminary results and review of the literature. Cornea. 2017;36(6):637-641. doi:10.1097/ICO.0000000000001202

83. Bleyen I, Saelens IEY, van Dooren BTH, van Rij G. Spontaneous corneal clearing after Descemet's stripping. Ophthalmology. 2013;120(1):215. doi:10.1016/j.ophtha.2012.08.037

84. Koenig SB. Planned descemetorhexis without endothelial keratoplasty in eyes with fuchs corneal endothelial dystrophy. Cornea. 2015;34 (9):1149-1151. doi:10.1097/ICO.0000000000000531

85. Moloney G, Chan U-T, Hamilton A, Zahidin AM, Grigg JR, Devasahayam RN. Descemetorhexis for Fuchs' dystrophy. Can J Ophthalmol. 2015;50(1):68-72. doi:10.1016/j.jcjo.2014.10.014

86. Borkar DS, Veldman P, Colby KA. Treatment of fuchs endothelial dystrophy by descemet stripping without endothelial keratoplasty. Cornea. 2016;35(10):1267-1273. doi:10.1097/ICO.0000000000000915

87. Huang MJ, Kane S, Dhaliwal DK. Descemetorhexis without endothelial keratoplasty versus DMEK for treatment of fuchs endothelial corneal dystrophy. Cornea. 2018;37(12):1479-1483. doi:10.1097/ICO.0000000000001742

88. Macsai MS, Shiloach M. Use of topical rho kinase inhibitors in the treatment of fuchs dystrophy after descemet stripping only. Cornea. 2019;38 (5):529-534. doi:10.1097/ICO.0000000000001883

89. Davies E, Jurkunas U, Pineda R. Predictive factors for corneal clearance after descemetorhexis without endothelial keratoplasty. Cornea. 2018;37(2):137-140. doi:10.1097/ICO.0000000000001427

90. Ruberti JW, Zieske JD. Prelude to corneal tissue engineering - gaining control of collagen organization. Prog Retin Eye Res. 2008;27(5):549577. doi:10.1016/j.preteyeres.2008.08.001

91. Lynch AP, Ahearne M. Strategies for developing decellularized corneal scaffolds. Exp Eye Res. 2013;108:42-47. doi:10.1016/j. exer.2012.12.012

92. Yin H, Qiu P, Wu F, et al. Construction of a corneal stromal equivalent with SMILE-derived lenticules and fibrin glue. Sci Rep. 2016;6:33848. doi: $10.1038 /$ srep33848

93. Fernández-Pérez J, Kador KE, Lynch AP, Ahearne M. Characterization of extracellular matrix modified poly( $\varepsilon$-caprolactone) electrospun scaffolds with differing fiber orientations for corneal stroma regeneration. Mater Sci Eng C Mater Biol Appl. 2020;108:110415. doi:10.1016/j. msec.2019.110415

94. Xu W, Wang Z, Liu Y, et al. Carboxymethyl chitosan/gelatin/hyaluronic acid blended-membranes as epithelia transplanting scaffold for corneal wound healing. Carbohydr Polym. 2018;192:240-250. doi:10.1016/j.carbpol.2018.03.033

95. Wu Z, Kong B, Liu R, Sun W, Mi S. Engineering of corneal tissue through an aligned PVA/collagen composite nanofibrous electrospun scaffold. Nanomaterials. 2018;8(2):124. doi:10.3390/nano8020124

96. Liao JK, Seto M, Noma K. Rho kinase (ROCK) inhibitors. J Cardiovasc Pharmacol. 2007;50(1):17-24. doi:10.1097/FJC.0b013e318070d1bd

97. Okumura N, Ueno M, Koizumi N, et al. Enhancement on primate corneal endothelial cell survival in vitro by a ROCK inhibitor. Invest Ophthalmol Vis Sci. 2009;50(8):3680-3687. doi:10.1167/iovs.08-2634

98. Okumura N, Koizumi N, Ueno M, et al. Enhancement of corneal endothelium wound healing by Rho-associated kinase (ROCK) inhibitor eye drops. Br J Ophthalmol. 2011;95(7):1006-1009. doi:10.1136/bjo.2010.194571

99. Okumura N, Koizumi N, Kay EP, et al. The ROCK inhibitor eye drop accelerates corneal endothelium wound healing. Invest Ophthalmol Vis Sci. 2013;54(4):2493-2502. doi:10.1167/iovs.12-11320 
100. Koizumi N, Okumura N, Ueno M, Nakagawa H, Hamuro J, Kinoshita S. Rho-associated kinase inhibitor eye drop treatment as a possible medical treatment for Fuchs corneal dystrophy. Cornea. 2013;32(8):1167-1170. doi:10.1097/ICO.0b013e318285475d

101. Proulx S, Brunette I. Methods being developed for preparation, delivery and transplantation of a tissue-engineered corneal endothelium. Exp Eye Res. 2012;95(1):68-75. doi:10.1016/j.exer.2011.06.013

102. Numa K, Imai K, Ueno M, et al. Five-year follow-up of first 11 patients undergoing injection of cultured corneal endothelial cells for corneal endothelial failure. Ophthalmology. 2021;128(4):504-514. doi:10.1016/j.ophtha.2020.09.002

103. Faye PA, Poumeaud F, Chazelas P, et al. Focus on cell therapy to treat corneal endothelial diseases. Exp Eye Res. 2021;204:108462. doi:10.1016/j.exer.2021.108462

104. Parekh M, Romano V, Hassanin K, et al. Biomaterials for corneal endothelial cell culture and tissue engineering. J Tissue Eng. 2021;12:204173142199053. doi:10.1177/2041731421990536

105. Zarouchlioti C, Sanchez-Pintado B, Hafford Tear NJ, et al. Antisense therapy for a common corneal dystrophy ameliorates TCF4 repeat expansion-mediated toxicity. Am J Hum Genet. 2018;102(4):528-539. doi:10.1016/j.ajhg.2018.02.010

106. Hu J, Rong Z, Gong X, et al. Oligonucleotides targeting TCF4 triplet repeat expansion inhibit RNA foci and mis-splicing in Fuchs' dystrophy. Hum Mol Genet. 2018;27(6):1015-1026. doi:10.1093/hmg/ddy018

107. ProQR Therapeutics. QR-504a for TCF4 mediated Fuchs endothelial corneal dystrophy. Available from: https://www.proqr.com/qr-504a-forfuchs-endothelial-corneal-dystrophy. Accessed July 6, 2021.

108. ProQR Therapeutics. Clinical trial for Fuchs endothelial corneal dystrophy. Available from: https://www.proqr.com/fuchs-focus-study-for-fuchsendothelial-corneal-dystrophy. Accessed July 6, 2021.

Clinical Ophthalmology

Dovepress

\section{Publish your work in this journal}

Clinical Ophthalmology is an international, peer-reviewed journal covering all subspecialties within ophthalmology. Key topics include: Optometry; Visual science; Pharmacology and drug therapy in eye diseases; Basic Sciences; Primary and Secondary eye care; Patient Safety and Quality of Care Improvements. This journal is indexed on PubMed Central and CAS, and is the official journal of The Society of Clinical Ophthalmology (SCO). The manuscript management system is completely online and includes a very quick and fair peer-review system, which is all easy to use. Visit http://www. dovepress.com/testimonials.php to read real quotes from published authors.

Submit your manuscript here: https://www.dovepress.com/clinical-ophthalmology-journal 